\title{
Hemomediastino por rotura de aneurisma bronquial
}

\section{Hemomediastinum caused by rupture of a bronchial artery aneurysm}

\author{
E. Martínez, I. Villar, S. Pérez, M. León, T. Rubio, C. García
}

\section{RESUMEN}

El hemomediastino por rotura de aneurisma bronquial es una patología muy poco frecuente. La presentación clínica puede ser variable, pudiendo simular patología aórtica y ser diagnosticado en situaciones de emergencia. La tomografía computarizada (TC) de tórax es la prueba de imagen inicial para el diagnóstico.

Se presenta el caso clínico de un varón de 70 años con clínica inicial de dolor torácico transfixivo que simulaba patología aórtica aguda. Se realiza TC con diagnóstico de sospecha de neoplasia mediastínica (Unidades Hounsfield/UH 38 - tejidos blandos) realizándose toma de biopsia por gastroscopia y broncoscopia con citología hemorrágica, presentando leve anemización al ingreso y permaneciendo hemodinámicamente estable y asintomático. Ante alta sospecha clínica de patología arterial se repite TC donde se describe hemomediastino en resolución confirmándose posteriormente la existencia de rotura de aneurisma bronquial mediante angiografía y procediendo a su embolización terapéutica.

Palabras clave. Hemomediastino. Arteria bronquial. Aneurisma.

\begin{abstract}
Hemomediastinum caused by rupture of a bronchial artery aneurysm is a very infrequent pathology. Clinical presentation can be variable; it can resemble an aortic pathology and be diagnosed in emergency situations. Computerized tomography (CT) of the thorax is the initial test for diagnosis.

We present the clinical case of a 70-year-old man with an initial clinical assessment of transfixive thoracic pain resembling an acute aortic pathology. TC was carried out with a diagnosis of suspected mediastinal neoplasia (Hounsfield Units/HU 38 - soft tissue), biopsy sampling was carried out by gastroscopy and broncoscopy with haemorrhagic pap smear, presenting light anemization on admission and remaining haemodynamically stable and asymptomatic. Facing a high clinical suspicion of arterial pathology, CT was repeated in which hemomediastinum was described in resolution, with subsequent confirmation of the existence of ruptured bronchial artery aneurysm through angiography and proceeding to therapeutic embolization.
\end{abstract}

Key words. Hemomediastinum. Bronchial artery. Aneurysm.
Servicio de Medicina Interna. Complejo Hospitalario de Navarra. Pamplona (Navarra)

Recepción: 2 de octubre de 2012

Aceptación provisional: 18 de octubre de 2012

Aceptación definitiva: 23 de noviembre de 2012

\section{Correspondencia:}

Elisabel Martínez Litago

Servicio de Medicina Interna.

Complejo Hospitalario de Navarra

C/ Irunlarrea, 3

31008 Pamplona (Navarra)

Email: elisamartinezlitago@hotmail.com 


\section{INTRODUCCIÓN}

El hemomediastino es una patología poco frecuente que puede deberse a diferentes causas siendo las más frecuentes secundaria a traumatismo o patología de aorta torácica. La clínica va a depender del origen y la cuantía del sangrado, siendo la más frecuente el dolor torácico y disnea. En ocasiones puede llegar a un estado de shock hipovolémico o hemodinámico por taponamiento mediastínico, siendo diagnosticado en situaciones de emergencia simulando disección de aorta o hemoptisis masiva. La tomografía computarizada (TC) de tórax es la prueba de imagen indicada para confirmar la presencia de hemomediastino y la causa desencadenante $e^{1,2}$.

La ruptura de aneurisma de arteria bronquial es una causa muy poco frecuente de hemomediastino, con menos de 100 casos descritos en la literatura ${ }^{3}$.

\section{CASO CLÍNICO}

Varón de 70 años con antecedentes personales de adenocarcinoma de próstata hace 7 años (T2a-b N0 M0), tratado con bloqueo androgénico, radioterapia y braquiterapia. Intervenido quirúrgicamente de apendicectomía y amigdalectomía. Sin toma de medicación habitual, como antecedentes familiares relevantes presenta hermana afecta de neoplasia de mama y hermano con neoplasia de pulmón.

Acudió al servicio de Urgencias por cuadro de dolor torácico transfixivo de inicio súbito asociado a cortejo vegetativo mientras realizaba labores agrícolas. Durante su estancia en el servicio de Urgencias permaneció asintomático y hemodinámicamente estable con TA 133/74 y FC $64 \mathrm{lpm}$. A la exploración presentaba palidez mucocutánea, auscultación cardiaca con tonos apagados rítmicos sin soplos, siendo el resto de la exploración normal. En la analítica inicial presentó hemograma normal con bioquímica y parámetros de coagulación anodinos, Dímero D $189 \mathrm{ng} / \mathrm{ml}$ (0-275), Péptido natriurético tipo B $80 \mathrm{pg} / \mathrm{ml}(0-100)$, troponina $0,008 \mathrm{ng} / \mathrm{ml}(0-0,04)$. En la radiografía $(\mathrm{RX})$ de tórax inicial presentaba ensanchamiento mediastínico (Fig. 1) por lo que se realizó TC urgente donde se describió masa prevertebral (Unidades Hounsfield/UH 38 - tejidos blandos), retrotraqueal, englobando el esófago y desplazando posteriormente la aorta la cual presentaba una morfología dentro de la normalidad, con discreto derrame pleural bilateral, siendo el resto de estructuras normales (Fig., 2). Ante los hallazgos de la prueba de imagen ingresó inicialmente en el servicio de Aparato Digestivo donde se llevó a cabo ecoendoscopia visualizando lesión heterogénea posterior a esófago sin depender de él, tomando biopsia con resultado negativo para células neoplásicas con citología hemorrágica. Se procedió a la realización de una broncoscopia, describiéndose compresión extrínseca a nivel de pared posterior de tercio distal de tráquea y bronquios principales realizando 3 punciones con salida de abundante material hemorrágico, con citología hemorrágica sin células neoplásicas. Se planteó realizar toracotomía para obtención de muestras. Ante la ausencia de hallazgos en las pruebas anteriormente realizadas, previa a la cirugía, se realizó interconsulta al servicio de Medicina Interna, quien revisando la historia clínica desde el ingreso objetivó anemización en las primeras horas de ingreso $(\mathrm{Hb} 14,1 \mathrm{~g} / \mathrm{dl}$ ) y posteriormente un valor de $\mathrm{Hb}$ de 11,2 g/dl permaneciendo estable. En nueva RX de tórax se observó desaparición del ensanchamiento mediastínico con mínima persistencia de derrame pleural. Se llevó a cabo toracocentesis diagnóstica con resultado negativo para neoplasia con citología hemorrágica. Dada la clínica inicial y los resultados de citología hemorrágica y ante la alta sospecha de complicación vascular a nivel torácico se solicitó nuevo TC siendo informado como pequeña imagen de aspecto hipodenso y homogéneo con un calibre aproximado de $1,5 \mathrm{~cm}$ compatible con hematoma en mediastino posterior en resolución. Se realizó angiografía donde se observó dilatación pseudoaneurismática de arteria bronquial en su origen, procediéndose a su embolización terapéutica sin incidencias.

\section{DISCUSIÓN}

La causa más frecuente de hemomediastino es el traumatismo previo seguido por ruptura o disección de aneurisma de aorta torácica ${ }^{1}$. La hemorragia espontánea en el mediastino puede deberse a coagulopatía, toma de anticoagulación oral o administración de heparina de bajo peso molecular, sangrado de masa mediastínica tumoral tal como timoma, teratoma, angiosarcoma, metástasis, adenoma paratiroideo, quiste tiroideo...1,2. El mecanismo de la hemorragia espontánea puede estar en relación con el aumento de presión intratorácica o por hipertensión arterial man- 


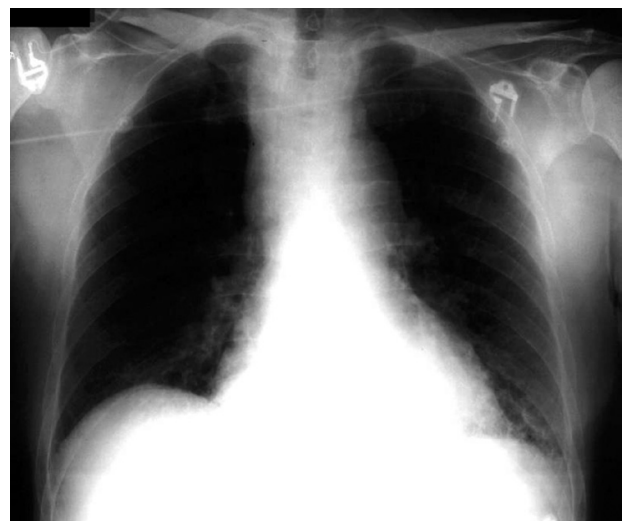

Figura 1. Radiografía simple de tórax donde se observa ensanchamiento mediastínico sin otras alteraciones patológicas significativas.

tenida. El hemomediastino secundario a ruptura de aneurisma de arteria bronquial es una causa muy poco frecuente y parece ser que el diámetro del aneurisma no es un factor de riesgo para su ruptura ${ }^{4,5}$. El aneurisma de arteria bronquial es un hallazgo poco frecuente, observándose en un $1 \%$ de los arteriogramas bronquiales ${ }^{3,4}$. Su etiología no está establecida. Se postulan varios factores predisponentes en la formación de aneurismas como son el aumento de flujo sanguíneo, aumento de presión sobre arterias bronquiales, lesión sobre pared arterial y pared vascular debilitada ${ }^{2}$. El origen puede ser congénito en relación a secuestro pulmonar o agenesia pulmonar, o adquirido en relación a inflamación crónica pulmonar (infecciones como tuberculosis, neoplasias), bronquiectasias, aterosclerosis... siendo el primer caso descrito en 1930 en una paciente con diagnóstico de silicosis ${ }^{3,4,6,7}$. Se han publicado casos en relación a causas menos frecuentes como son alteraciones vasculares sistémicas como la enfermedad de Rendu-Osler-Weber o aneurismas micóticos, o debido al aumento de presión en arteria bronquial generada por ganglios linfáticos mediastínicos en la sarcoidosis ${ }^{3,5,7,8}$. Aun así en muchas situaciones la etiología sigue siendo desconocida.

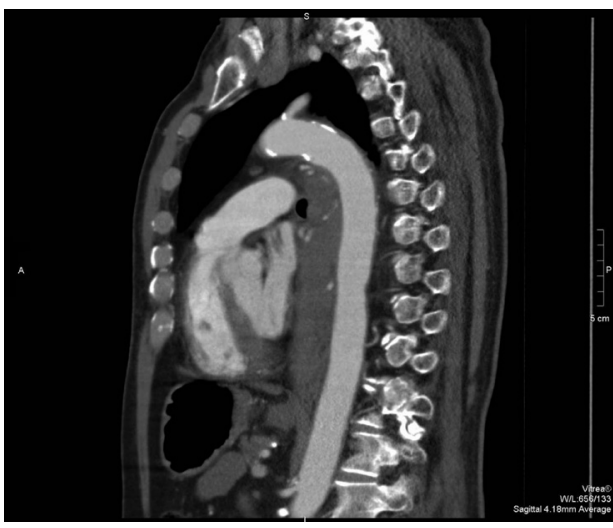

Figura 2 Tomografía computerizada donde se describe masa prevertebral retrotraqueal, desde D2 a D12, englobando el esófago y desplazando posteriormente la aorta la cual presenta una morfología dentro de la normalidad.

En nuestro caso clínico el factor desencadenante puede estar en relación al aumento de presión intratorácica por el esfuerzo físico realizado.

Los aneurismas de la arteria bronquial se clasifican por su localización anatómica intrapulmonar o en mediastino debido a sus diferentes formas de presentación clínica.

Permanecen asintomáticos hasta su presentación clínica tras su ruptura o por compresión extrínseca de estructuras adyacentes ${ }^{7}$. En ocasiones el diagnóstico es incidental por la realización de radiografía de tórax ${ }^{3}$. Se han publicado dos casos en los que la sospecha inicial era de patología tumoral esofágica al igual que en nuestro caso, tras la realización de esofagograma, en uno de ellos por clínica de disfagia ${ }^{3,5}$. En ambos se describió esofagograma alterado por compresión extrínseca de la mucosa esofágica. En uno de ellos se confirmó la presencia del aneurisma de arteria bronquial tras realización de TC con contraste3. El otro caso requirió la realización de toracotomía ya que en el TC presentaba una densidad de $47 \mathrm{UH}$ (tejidos blandos) que al igual que en nuestro caso era sugestiva de patología tumoral dependiente de esófago ${ }^{5}$.

Los aneurismas de localización intrapulmonar se presentan habitualmente como 
hemoptisis masiva o intermitente e incluso imitando a un tumor endobronquial. Los aneurismas que se localizan en el mediastino pueden presentarse como masa mediastínica, síndrome de vena cava superior, hemomediastino, disfagia o como hematemesis por erosión de la pared esofágica ${ }^{3}$. Pueden producir dolor torácico, epigástrico o de espalda con o sin shock hemodinámico simulando disección o rotura de aorta torácica como ocurre en el caso presentado en este trabajo.

Ante un paciente con sospecha de hemomediastino debe realizarse una TC con el fin de conocer el punto de sangrado. En situación hemodinámicamente estable debe confirmarse el diagnóstico mediante angiografía arterial bronquial para obtener más detalles sobre la situación y relación anatómica con estructuras subyacentes ${ }^{4}$.

Todo aneurisma de arteria bronquial debe ser tratado al diagnóstico independientemente de la clínica debido al alto riesgo de sangrado con compromiso vi$\mathrm{tal}^{3,7}$. El tratamiento depende de la etiología y de la afectación hemodinámica y respiratoria ${ }^{2}$.

El tratamiento habitual en situación hemodinámica estable es el tratamiento endovascular mediante embolización transcatéter local ${ }^{9,10}$. Las complicaciones son poco frecuentes, dándose en $1 \%$ de los casos, como infarto medular, perforación esofágica, dolor torácico, fiebre ${ }^{2} .$. La cirugía está indicada en aquellos pacientes con contraindicaciones para la embolización (Ej. alergia al contraste iodado) con cirugía mediante ligadura o resección.

\section{BIBLIOGRAFÍA}

1. Ruiz-Salas A, Navarro-Arce I, Morcillo-Hidalgo L, Melero-Tejedor JM. Hemomediastino es- pontáneo y taponamiento cardíaco extrapericárdico en relación con heparina de bajo peso molecular. Rev Clin Esp 2013; 213: 6869.

2. Quero-Valenzuela F, Piedra-Fernández I, SevillaLópez S, Cueto-Ladrón de Guevara A. Spontaneous hemomediastinum and hemotórax alter dissecting bronchial artery aneurysm. Interact CardioVasc Thorac Surg 2011; 12: 619-621.

3. Tanaka K, Ihaya A, Horiuci T, Morioka K, Kimura T, UESAKA T et al. Giant mediastinal bronchial artery aneurysm mimicking benign esophageal tumor: a case report and the review of 26 cases from literature. J Vasc Surg 2003; 38: 1125-1129.

4. Mizuguchi S, Inoue K, Kida A, Isota M, Hige K, AOYAMA T AND IsHIKAWA T. Ruptured bronchial artery aneurysm associated with bronchiectasis: a case report. Ann Thorac Cardiovasc Surg 2009; 15: 115-118.

5. Tringali S, Tiffet O, Berger J, Culleeret J. Bronchial artery aneurysm disguised as a leiomyoma of tha esophagus. Ann Thorac Surg 2002; 73: 632-633.

6. De Dominicis F, Leborgne L, Raymond A and Berna P. Right pulmonary artery agenesis and coronary-to-bronchial artery aneurysm. Interact CardioVasc Thorac Surg 2011; 12: 507-510.

7. Hu CX, Huang S, Xu ZW, Chen W, Huang JS and Fu Z. Combination of aortic stent-graft and arterial embolization for ruptured bronchial artery aneurysm. Ann Thorac Surg 2011; 92: 19-21.

8. Suen HC, DuMontier CC, Boeren J, Charland W, DAILY BB. Ruptured bronchial artery aneurysm associated with sarcoidosis. J Thorac Cardiovasc Surg 2003; 125: 1153-1154.

9. Lococo F, Cafarotti S, Margaritora S, Granone P. Hemomediastinum and hemothorax after dissecting bronchial artery aneurysm: the limit of the surgical option. Interact Cardiovasc Thorac Surg 2011; 12: 621.

10. Hung SW. Where is the ruptured bronchial artery aneurysm? Eur J Cardiothorac Surg 2010; 38: 389-390. 